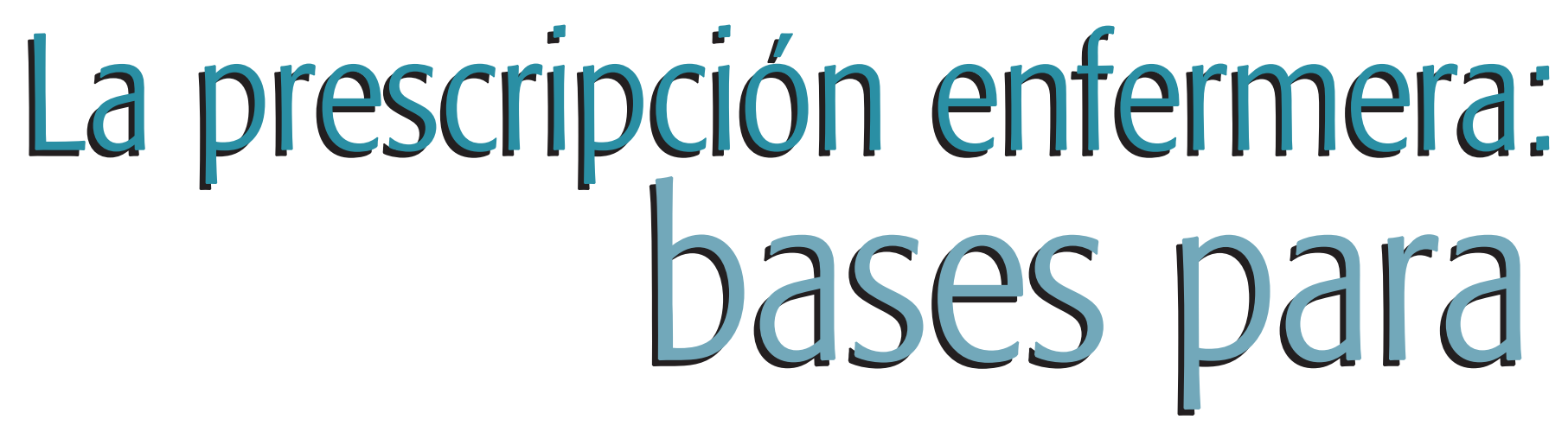

NÚRIA CUXART AINAUD

Directora de programas. Col-legi Oficial d'Infermeria de Barcelona. Barcelona. España.

Este artículo es resultado de la conferencia "La prescripción

enfermera: una situación indispensable para la mejora del sistema de salud", que se impartió el 29 de junio de 2006 por invitación del Colegio Oficial de Diplomados en Enfermería de Guipúzcoa en la Jornada "La prescripción farmacológica enfermera: análisis de la situación y futuro" organizada en el marco de los XXV Cusos de Verano/Uda Ikastaroak y los XVIII Cursos Europeos/Europar Ikastaroak organizados por la Universidad del País Vasco, Gobierno Vasco, Diputación Foral de Guipúzcoa y Ayuntamiento de San Sebastián.

EL TRABAJO, QUE RECOGE la llamada "propuesta catalana" o "propuesta de Barcelona" para la prescripción enfermera, pone énfasis y prioriza la prescripción autónoma (también llamada independiente) de la enfermera* frente a la incluida en el texto de la Ley de garantías y uso racional del medicamento aprobada en las Cortes Generales el mismo día que se dictaba la conferencia que ha originado este artículo.

* En este artículo se utiliza el término enfermera para designar a los profesionales de ambos sexos, y para diferenciarlos de otros profesionales de enfermería, como los auxiliares de enfermería, que no tienen capacidad prescriptora.
Se pretende, pues, argumentar la necesidad de reconocer la capacidad prescriptora de las enfermeras en aquellas técnicas, productos y fármacos que utilizan en el desarrollo de sus competencias. Es decir, defender la prescripción en el ámbito competencial en el que la enfermera es autónoma -en el desarrollo de la función de cuidar-, en el basado en un modelo de cuidados donde las prescripciones que realiza la enfermera son de su exclusiva responsabilidad como única vía posible $\mathrm{y}$ en donde las enfermeras pretenden conseguir el reconocimiento legal.
Para ello, el artículo se ha estructurado en coherencia con los 3 objetivos que pretende alcanzar, y que son los siguientes:

1. Presentar las razones en las que - a mi modo de ver y de la corporación profesional que represento- se fundamenta la necesidad de desarrollar la capacidad de prescripción de la enfermera.

2. Definir la prescripción enfermera y revisar las diferentes opciones que se nos presentan para su desarrollo.

3. Poner a su consideración los motivos a favor del "sí" y los motivos a favor del "no" al desarrollo de esta capacidad prescriptora.

\section{MARCO GENERAL}

Para afrontar la cuestión de la prescripción, como en muchísimas ocasiones ante cualquier otra cuestión relacionada con la práctica enfermera, me veo obligada a partir de la premisa de que -a mi modo de ver, y cada vez estoy más convencida de ello- la dificultad ante la que nos encontramos las enfermeras es que nos movemos en un entorno extraño, confuso, en donde es necesario intervenir con urgencia.

El concepto actual de salud y el modelo de atención para la salud que contemplan las diversas normativas -mundiales, europeas, estatales o 
locales- regulan y desarrollan los sistemas sanitarios, basándose en unos conceptos de persona, entorno y salud que son integrales, globalizadores, dinámicos y que determinan un modelo de atención para la salud centrado básicamente en la promoción, educación, prevención y readaptación y no, como ha venido sucediendo hasta ahora, exclusivamente en la curación de la enfermedad, y son muy cercanos al objetivo profesional de las enfermeras.

Pero el caso es que no se ha conseguido alcanzar todavía el nivel de desarrollo deseado en la práctica cotidiana ${ }^{1}$.

Aunque ni mucho menos el más reconocido, éste es uno de los principales problemas al que ahora mismo se enfrentan la atención sanitaria y social en nuestro país, situación que las enfermeras deberíamos analizar en profundidad y ser capaces de convertirla en oportunidad e intentar liderarla. Me refiero a la atención a las personas en situación de dependencia, grupos vulnerables, todo tipo de abordaje grupal, la lucha contra el medicocentrismo, la autonomía de las personas, etc.

Pero la realidad es que no acaban de concretarse objetivos que se puedan asumir desde diferentes dimensiones del propio "cuidar", y la realidad es también que las enfermeras no acabamos de encontrar soluciones realmente efectivas a problemas históricamente planteados (la invisibilidad, la desvalorización, etc.) y a los que rodean nuestra práctica asistencial y que la influyen fuertemente (como la precariedad laboral, la complejidad de la demanda y un largo etcétera). No hablaré de ello, entre otros motivos, porque creo que, en gran parte, éstos no son los problemas sino las consecuencias de uno más general y que intentaré analizar.

Ante el reto de tomar partido en la cuestión de la prescripción enfermera, como en tantas y tantas otras cuestiones que afectan tanto a la práctica como al desarrollo general de nuestra profesión, las enfermeras acostumbramos a situarnos en un terreno dicotómico que nos lleva, a mi modo de ver erróneamente, a pensar que debemos escoger entre dos posibilidades:

- La vía "corporativista", en el peor sentido de la palabra (el afán de un grupo profesional para hacer prevalecer los intereses del mismo frente a los de la sociedad a la que sirve).

- La vía de servicio a las personas que atendemos, la familia y la comunidad sin preguntarse nada más: ni su pertinencia, ni sus consecuencias, ni incluso su oportunidad.

Nos movemos, pues, entre la alternativa de "lo que es bueno" para las personas que atendemos y "lo que es bueno" para la profesión, como si de los extremos del recorrido de un péndulo se tratara, como si una vía tuviese que excluir necesariamente a la otra y preguntándonos constantemente cuál de ellas debemos escoger. Les propondré, también, una solución para salir de esta duda que a mí me ha resultado extremadamente útil.

Esta situación dicotómica -y siempre a mi modo de ver- ha dañado gravemente e históricamente a la profesión, olvidando que, en realidad, las profesiones las configuran una serie de elementos sin los cuales no pueden considerarse como tal. Sé que pensarán ustedes que no es hoy el día de hablar de ello, pero no puedo dejar de dedicar, aunque sólo sean unos minutos, a esta cuestión refiriéndome al servicio a las personas y el desarrollo de la profesión.

\section{EL SERVICIO A LAS PERSONAS Y EL DESARROLLO DE LA PROFESIÓN}

\section{El servicio a las personas}

Citando a Ehrenreich, dice Rosamaría Alberdi $^{2}$, enfermera a la que yo me refiero muchas veces por el altísimo conocimiento que tiene de las circunstancias que rodean a la profesión enfermera, sus características y las consecuencias de su actuación: "Una profesión obtiene y mantiene su posición gracias a la protección y al patrocinio de 


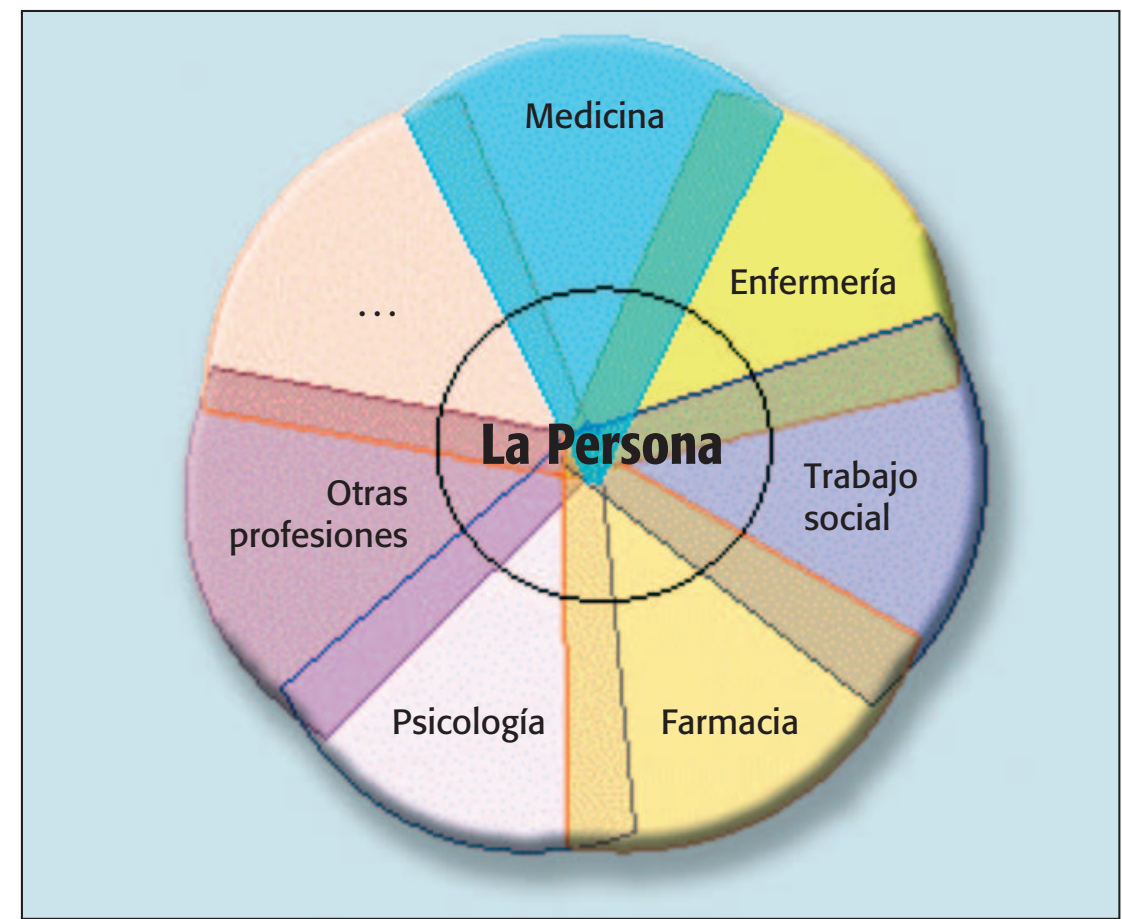

Fig. 1. Aportación de las profesiones de la salud a la atención de la persona o la comunidad.

la sociedad que ha llegado al convencimiento de que su trabajo ofrece algún interés especial".

Desde una perspectiva sociológica, se identifica a una profesión tanto por el grado de conocimientos particulares como por las características de los servicios que presta la/el profesional. Así, las enfermeras y la enfermería como profesión, existen porque también existen unas necesidades que atender, unos problemas que resolver, y es a las enfermeras (y por lo tanto a la profesión en su conjunto) a quienes les corresponde aportar soluciones específicas, basándose en unos conocimientos y a unas habilidades concretas.

Es decir, el trato consiste en que la profesión enfermera ofrece unas acciones profesionales, reconocidas como necesarias, y, como retribución, la sociedad paga un determinado precio, individual y colectivo (educación universitaria, normas legales que permiten su ejercicio, puestos de trabajo y valoración social). Y esto está establecido así. Y o se quiere o no se quiere ser profesional. Y si se quiere, esto es lo que hay.

$\mathrm{Y}$ este servicio tiene un contenido técnico (competencias y capacidades de la profesión) y un carácter ético (de compromiso y de respuesta a necesidades con la garantía de esta realización), lo que obliga a reexaminar constantemente las capacidades y a mantenerlas en el nivel exigido. Y para ello, también las profesiones mantienen un código ético que obliga a sus componentes, en conciencia y de forma corporativa, y que además permite garantizar a la sociedad las medidas que la propia profesión determine en caso de incumplimiento del compromiso profesional.

Y también proporciona el apoyo legal a este servicio. Es decir, el reconocimiento explícito de la capacidad de actuar en determinadas ocasiones y circunstancias del ejercicio de la profesión. En España, esta capacidad para actuar está reconocida por la propia titulación universitaria, la Ley de Ordenación de las Profesiones Sanitarias, la de Cohesión y de Calidad del Sistema Nacional de Salud y el Estatuto de la profesión aprobado por Real Decreto (1231/2001), que han configurado a la enfermería como una profesión con autonomía, funciones y responsabilidades propias.

En todo caso, el servicio ha de estar perfectamente definido: cuál es la aportación concreta que esta disciplina profesional puede hacer para la solución de esas necesidades de cuidado planteadas, cuáles son estas necesidades y qué situaciones de competencia conjunta de varios profesionales hay.

Otra vez hace falta pararnos aquí para recordar que es exactamente aquí donde recae "la madre de todos los males".

"Madre de todos los males" que, aunque lo he hecho en multitud de ocasiones, no me resisto a utilizar una imagen que creo que la puede representar (fig. 1).

Para explicar de forma más gráfica este concepto, en la figura he representado en un círculo central al sujeto de nuestros cuidados y de color azul, la visión que hasta ahora ha prevalecido (todavía prevalece) de los problemas que presentan las personas. Si miramos, pues, la medicina, identificaremos problemas (enfermedades) que corresponden a la medicina y que, por tanto, son responsabilidad del médico.

Si miramos la enfermería, es decir, observar a la persona o al conjunto de la sociedad a través del filtro de la disciplina enfermera (constituida por conocimientos, habilidades y principios éticos), este concepto introducido por Alberdi y al que ya denominamos de manera general "la mirada enfermera" destaca una porción (de color amarillo) formada por las situaciones y problemas que las enfermeras identificamos y podemos ayudar a resolver y que constituyen nuestro objetivo profesional. Efectivamente, hay una zona de confluencia, que corresponde a problemas casi idénticos y que podremos resolver desde la medicina o desde la enfermería y que, por tanto, lo harán tanto médicos como enfermeras. De hecho, si revisáis nuestras clasificaciones diagnósticas, las encontraréis (el dolor, la fatiga, la ansiedad, la diarrea, el estreñimiento, la hipertermia, el deterioro de la integridad cutánea o tisular, etc.).

Desde esta percepción única se concreta nuestra aportación profesional y la necesidad de provisión de cuidados enfermeros, a lo que debemos dedicar todos los esfuerzos porque constituye nuestro objetivo profesional. Y por muchas otras razones, pero 2 fundamentales y que describiré a continuación. 
La primera, porque, si no lo hacemos de este modo, desatendemos aquellas situaciones, necesidades y problemas que las enfermeras, cuando "miramos desde la enfermería" a la persona, a la familia y a la comunidad, podemos determinar, resolver o mejorar. Y esto es un fraude, y se deberá solucionar. Como sucede si se obvia también la mirada de otros profesionales. Y es un fraude porque, de quedarnos (o de querer ocupar) las enfermeras en la porción de atención que se deriva de la atención médica, privamos a las personas que atendemos de una mayor porción de posibilidades para su atención y porque una gran parte del servicio que nos ha encomendado no se lo proporcionamos, y esto es una desatención. Desatención de la que bien es verdad, después de veinticinco años de denunciarla, ya no es sólo responsable la enfermera (que batalla muchas veces individual y colectivamente contra ella), sino el conjunto de las estructuras responsables de la atención de salud y social y que erróneamente identificamos a través de la palabra "sociedad".

Siento ser tan dura en este sentido, pero es que de lo que estamos hablando es de una profesión, de un compromiso, de una responsabilidad para con la sociedad que se le ha encomendado (a la profesión están ocupadas, y o "vamos a la guerra" (que no es el caso) para disputárnoslas con otros profesionales o vamos de "realquilados" en la parcela de otro (pero el propietario siempre será el otro). Es decir, o nos situamos en la parcela "enfermera" (de la enfermería) o no hay desarrollo profesional.

Hubo tiempos en que se dedicaron muchos esfuerzos a ello, y es necesario recordar que el compromiso profesional está indisolublemente unido a la observancia de unas bases disciplinares que lo sustentan y, cuando éste se debilita, es la propia profesión la que se ve debilitada en su legitimidad social. Sin ello no es posible una prestación altamente diferenciada y cualificada, y sin prestación diferenciada no hay contrato social.

Esto del "cuidar" y del "cuidado" no consiste en "algo" que, como se supone que ya lo aprendimos un día y ya nos lo sabemos, podemos olvidar.

\section{El desarrollo de la profesión}

En palabras de la misma autora a la que me he referido anteriormente, "una profesión reconocida no es solamente un grupo de expertos que se autoproclaman como tales, sino una corporación que tiene autoridad legalmente reconocida
Si bien los 4 primeros los tenemos más o menos resueltos o como mínimo encaminados, en el quinto (autonomía, control y responsabilidad sobre nuestro campo de actuación) "pinchamos" estrepitosamente. Y este es un asunto pendiente importante porque afecta gravemente al reconocimiento social y al poder y a la identidad como profesión. Quizás otro día podríamos dedicarlo a las cuestiones que rodean al poder profesional, y yo me ofrezco encantada a establecer un debate para revisar de nuevo las causas, los caminos que hemos seguido, sus consecuencias y, sobre todo, todo aquello que deberíamos ir remediando ya.

Y es precisamente en este "disponer de autonomía, control y responsabilidad sobre su campo de actuación y sobre el método que utiliza para realizar su tarea, incluida su modificación", donde la profesión, a través de sus corporaciones, debe empezar a tomar decisiones ya.

La literatura dedicada al desarrollo de las profesiones coincide de manera general en que las 3 características centrales de las profesiones son: la base de un conocimiento especializado, el servicio y la autonomía profesional. He hecho referencia a la primera y a la segunda (al conocimiento específico

\section{El concepto actual de salud y el modelo de atención para la salud que contemplan las diversas} normativas -mundiales, europeas, estatales o locales- regulan y desarrollan los sistemas sanitarios, basándose en unos conceptos de persona, entorno y salud que son integrales, globalizadores, dinámicos y que determinan un modelo de atención para la salud centrado básicamente en la promoción, educación, prevención y readaptación y no, como ha venido sucediendo hasta ahora, exclusivamente en la curación de la enfermedad, y son muy cercanos al objetivo profesional de las enfermeras.

enfermera) y que por lo tanto confía en sus miembros (las enfermeras) para que lo lleven a cabo.

La segunda de las razones es porque impedimos nuestro propio desarrollo profesional. Pueden ustedes pensar que estas afirmaciones no constituyen, precisamente, una novedad. Cierto, pero de todos modos lo menciono porque, con independencia de mi convencimiento de que así, desde sólidas bases disciplinares, es como "debo funcionar", es que es sólo desde esta parcela donde podemos desarrollarnos como profesionales. Las otras parcelas ya para seleccionar a sus miembros y regular su práctica profesional"2.

Dice Alberdi que una profesión se caracteriza por tener un cuerpo de conocimientos propio, desarrollar la práctica de acuerdo con él, haber definido un código ético y actuar en consecuencia o poseer como objetivo de la actuación profesional cuestiones que significan necesidades o problemas para la comunidad. Y también de "disponer de autonomía, control y responsabilidad sobre su campo de actuación y sobre el método que utilice para llevar a cabo su tarea, incluida su modificación"4. y al servicio), por lo que sólo hay que recordar que la base de conocimiento de las profesiones confiere estatus y poder social. Pero sobre todo que esta base de conocimientos es una pieza central para la justificación de su autonomía, autonomía que se expresa también en 2 niveles:

- El individual, pues cada profesional controla su trabajo personal.

- El corporativo, puesto que sólo los miembros de la corporación disponen del conocimiento suficiente para determinar -con el máximo consenso 
posible-su ámbito de competencia, para juzgar la competencia de sus miembros y para regular el ejercicio profesional.

Y porque disciplina equivale a ejercicio de autorregulación, basándose en criterios éticos y cientificotécnicos que emanan del código (ético) de la profesión y del conocimiento específico que se supone monopoliza. La autorregulación constituye la quintaesencia de toda profesión, hasta tal punto que se ha llegado a afirmar que una profesión -a mi modo de ver y de la corporación profesional a la que represento- se fundamenta la necesidad de desarrollar la capacidad de prescripción de la enfermera, y para finalizar este primer apartado, se propone la solución para salir de la situación dicotómica en que creo nos situamos las enfermeras ante cualquier determinación o decisión.

Para ello, debo citar de nuevo a Rosamaría Alberdi ${ }^{7}$ y partir de su definición de otro de los elementos indispensables del desarrollo profesional

\section{Nos movemos, entre la alternativa de "lo que es bueno" para las personas que atendemos y "lo que es bueno" para la profesión, como si de los extremos del recorrido de un péndulo se tratara, como si una vía tuviese que excluir necesariamente a la otra y preguntándonos constantemente cuál de ellas debemos escoger.}

no es más que una ocupación que ha conseguido autorregularse $e^{5}$.

$\mathrm{Y}$ es por ello por lo que es a las profesiones - a cada una de ellas y sólo a ellas- a las que les corresponde la definición, la ampliación o la modificación de sus competencias, si bien corresponde a los órganos políticos y legislativos adecuar el marco legal a manera de reconocimiento y de regulación para hacerlo posible.

Y por eso hay que asumir también esta responsabilidad. Pero para todo ello hay una premisa básica que consiste en el compromiso con su objetivo fundamental, y esto sólo se consigue fortaleciendo y fortaleciéndose en las bases que sustentan nuestra profesión y comprometiéndose -profesionalmente hablando-, en el sentido también expresado por Alberdi de "contraer voluntariamente una responsabilidad como producto de la convicción íntima de que, así, se actúa con la responsabilidad profesional"6. Es decir, comprometiéndose con su objetivo profesional, que no es otro que la prestación de cuidados. Y si esto es así, una consecuencia clara: la prescripción enfermera o lo que sea, con todas las dificultades del mundo que nos podamos imaginar, será aquello que las enfermeras queramos que sea y nada más.

Analizadas pues, aunque muy rápidamente, las razones por las que de "trabajo excelente", que lo define como "aquel que cumple 2 condiciones: soluciona problemas de los clientes y hace evidente la contribución profesional al bienestar individual y social. A partir de esta definición -propone la autora-, se trata de hacer 2 cosas. La primera es utilizar 2 preguntas 'tozudas' (¿ayuda a solucionar problemas? y icontribuye a que se haga evidente la aportación enfermera?). Y en segundo lugar, se trata de actuar en consecuencia con la respuesta".

A mi modo de ver, pues, sólo será necesario aplicar este magnífico truco que consiste en formularse estas 2 preguntas que deberíamos plantearnos ante la duda de si estamos delante de una práctica excelente. Es decir, ante la duda de si se actúa o no de manera profesional excelente cuando dudamos ante la posibilidad de aceptar una propuesta con relación a la prescripción enfermera, es necesario hacerse estas preguntas tozudas: ¿Ayuda esto a solucionar problemas a las personas que atiendo? y ¿Contribuye al desarrollo de mi disciplina?

Si la primera es afirmativa y la segunda no (es decir, resuelve problemas a las personas que atiendo pero no contribuye al desarrollo disciplinar), cuidado con la decisión, porque probablemente estemos resolviendo problemas fuera de nuestro ámbito de responsabilidad. De lo contrario, si la primera es negativa y la segunda afirmativa (es decir, no resuelve problemas a las personas que atiendo aunque contribuye al desarrollo disciplinar), seguramente estemos ante una cuestión tan exclusivamente dentro del plano teórico que será necesario madurar. Pero si las 2 respuestas son afirmativas -dice Alberdi-, es decir, ayuda a solucionar problemas y contribuye a que se haga evidente mi aportación o, lo que es el mismo, ayuda a la construcción de la disciplina enfermera, querrá decir que no me he equivocado.

Y en base al resultado de estas 2 preguntas, que determinará si se actúa bajo criterios de trabajo excelente, en el caso que nos ocupa, es decir, si las enfermeras españolas debemos asumir la responsabilidad de la prescripción autónoma, la respuesta es que, efectivamente, la debemos afrontar. Y hablamos de prescripción autónoma (o independiente) sin ninguna duda, sin miedo y de la mejor manera posible, como un instrumento importante para nuestro ejercicio profesional porque resolverá problemas a las personas que atendemos y contribuirá al desarrollo profesional.

Resolverá problemas porque prescribirá para buscar la mejor técnica (p. ej., la de relajación o la de abordaje grupal). O buscará el mejor producto ante la incontinencia (urinaria y/o fecal) o el material más adecuado para la persona portadora de un estoma. $\mathrm{O}$ aquel fármaco básico para paliar el dolor o la fiebre. En definitiva, intervenir en aquellas situaciones donde ella (la enfermera) es quien mejor las puede manejar.

O dicho de otro modo, en todas aquellas cuestiones donde, una vez detectada la necesidad y determinado el recurso, el profesional encargado de autorizar el acceso al producto (en definitiva, la receta) es el que mejor información tiene sobre la decisión más correcta que hay que tomar. En tantas situaciones en las que habitualmente no es el médico quien las atiende, sino la enfermera y que, en cambio, no tiene autoridad formal de prescribir o recetar.

Y contribuirá al desarrollo profesional y disciplinar porque abrirá nuevas posibilidades que le permitirán plantear avances e innovaciones que resuelvan los interrogantes que se plantean en su práctica. Es decir, "la descripción de 
fenómenos, la búsqueda de explicaciones, la predicción de resultados que han de permitir a la enfermera avanzar profesionalmente" ${ }^{\text {. Y Y porque }}$ enfermeras dispuestas a interrogarse y a buscar respuestas desde una concepción disciplinar propia, desde una perspectiva diferenciada, fundamentarán las intervenciones profesionales enfermeras.

Y así, de este modo, las enfermeras y la profesión enfermera serán fuertes.

Porque serán capaces de convertir este conocimiento enfermero y la aplicación de este en servicios diseñados desde su ámbito competencial y contribuirán a convencer a las empresas y a los sistemas de salud y social de su, que no de otra, aportación profesional. Como tantas y tantas cosas, la profesión hace falta construirla y defenderla cada día. Y como apunta Guy ${ }^{9}$, los profesionales son diferentes de los no profesionales porque ellos están convencidos de que lo son y los demás así lo creen.

Y esta es la prescripción que yo defiendo para las enfermeras. La que se enmarca en sus competencias, la que resuelve problemas y la que contribuye a su desarrollo profesional. Esta "prescripción" que, además, no da ningún miedo a la enfermera. Porque, sobre estas situaciones, es quien sabe más.

Y hasta aquí creo haber alcanzado el primero de los objetivos, las razones por las que -a mi modo de ver y de la corporación profesional a la que represento- se fundamenta la necesidad de desarrollar la capacidad de prescripción de la enfermera. Y a partir de aquí, el segundo. Es decir, la prescripción enfermera y las diferentes opciones que se nos presentan para su desarrollo.

\section{LA PRESCRIPCIÓN ENFERMERA Y LAS DIFERENTES OPCIONES QUE SE NOS PRESENTAN PARA SU DESARROLLO}

Todos coincidimos en que la enfermera es quien satisface, junto con otros profesionales, las necesidades en materia de salud de los ciudadanos a través de la dispensación de cuidados enfermeros. Es también quien gestiona los recursos para que las personas puedan afrontar la enfermedad colaborando en la prevención, promoción, seguimiento, tratamiento, rehabilitación y acompañamiento en el proceso de duelo, garantizando en todo momento la continuidad asistencial.

\section{La definición de prescripción enfermera}

Según un estudio Delphi ${ }^{10}$ realizado, las enfermeras definen la prescripción enfermera como "emitir un criterio profesional sanitario dirigido al paciente para el que se propone la realización de una acción en base a un juicio clínico y terapéutico o de cuidados".

La Organización Colegial de Enfermería (Consejo General) la define como "la capacidad de seleccionar, guiados por criterios de buena práctica, diferentes materiales, productos y/o dispositivos encaminados a satisfacer las necesidades de salud del usuario y la población, apoyados por el juicio clínico enfermero y administrados en forma de cuidados".

El Col.legi Oficial d'Infermeria de Barcelona (COIB) entiende como prescripción enfermera "la capacidad de seleccionar e indicar medicamentos y productos sanitarios, en beneficio y satisfacción de las necesidades de salud de la persona y la población durante la administración de los cuidados, bajo criterios de buena práctica clínica y juicio
- Prescribir va más allá de la complementación de una receta para la dispensación de un producto; prescribir implica indicar el mejor régimen terapéutico frente a un problema de salud, con una valoración previa del problema, basado en el juicio clínico y enmarcado en unas competencias profesionales.

- Recetar, en el sentido de la cumplimentación y recogida de la receta, es un acto administrativo que, a través de la informatización y aplicación de las nuevas tecnologías, ya disfruta de avances significativos que facilitan su realización y que normalmente se expide para ser presentado en una farmacia para su adquisición.

Hay quien defiende la sinonimia de las 2 palabras, y no creo necesario en este momento entrar en una discusión semántica de la cuestión. En todo caso, mi objetivo es dejar claro, meridianamente claro, que la prescripción que yo defiendo, que en el COIB defendemos, tiene que ver principalmente con la que corresponde a nuestro rol autónomo, es decir, al de nuestra exclusiva responsabilidad. Y está claro que deberemos poder hacerlo también en el marco de rol de colaboración (enseguida veremos en qué consiste la propuesta "prescripción colaborativa")

\section{Según un estudio Delphi realizado, las enfermeras definen la prescripción enfermera como "emitir un criterio profesional sanitario dirigido al paciente para el que se propone la realización de una acción en base a un juicio clínico y terapéutico o de cuidados".}

clínico enfermero que le otorgan sus competencias".

En el caso de nuestra corporación preferimos añadir al verbo "seleccionar" el de "indicar", en el estricto sentido de la palabra por lo que se refiere a "mostrar o significar".

En todo caso, coincidimos todos en la necesidad de no confundir la diferencia entre los términos "recetar" y "prescribir", pues últimamente en algunos ámbitos -especialmente políticos- se mostraba cierta confusión respecto a 2 conceptos que no son sinónimos. Por lo tanto, es necesario aclarar que partimos de los conceptos de que: pero, en todo caso, la pregunta entonces reside en si estamos hablando de "prescribir/recetar" o sencillamente de un "administrar avanzado", que efectivamente ya realizamos en una situación de alegalidad y que, a mi modo de ver siempre después de resolver la prescripción autónoma, también deberemos regular.
Las diferentes opciones que se nos presentan para el desarrollo de la prescripción enfermera Según distintas informaciones proporcionadas por el Consejo General ${ }^{11}$, la prescripción enfermera es un término que se usa para describir más de un tipo 
de práctica en diferentes países, y existen 2 modelos en los que la enfermera puede participar en dicha prescripción:

- Prescripción independiente. En esta modalidad la enfermera realiza la valoración y el diagnóstico y prescribe libremente. En España, esta capacidad está reservada a médicos, veterinarios y odontólogos, aunque en algunos países las enfermeras están autorizadas a realizar prescripciones de un formulario cerrado, como es el caso del Reino Unido, donde en este momento se plantean abrirlo.

- Prescripción colaborativa (individualizada o estandarizada), a la que yo denomino "administración avanzada de medicación”. La enfermera puede "prescribir como colaboradora de un
De las bondades de los protocolos o las guías, entre otras para asegurar unos estándares mínimos de calidad, nadie puede dudar. Sin embargo, lo que no suele ser tenido en cuenta son los peligros potenciales que encierran para la práctica profesional. Uno de ellos, la importante limitación sobre la autonomía de actuación de la enfermera con consecuencias como la confrontación con otro profesional. Otro riesgo es que esta "normalización" supone muchos protocolos que se confeccionan siempre basándose en los postulados de la disciplina médica, desde su orientación, desde su mirada y no como resultado del acuerdo de 2 modos distintos de abordarlos. Por lo que entonces "el consensuar", en el sentido de "adoptar una decisión de común acuerdo entre otros profesionales y en la que estamos dispuestas a contribuir. Pero si "el sistema no quiere" (no está dispuesto a que ni tan sólo se regularice la que, de hecho y mayoritariamente, se hace), es al sistema al que le debe preocupar.

$\mathrm{O}$ en todo caso, eficiencia entendida como la producción de los bienes y servicios que la sociedad valora más, al menor coste posible o a la relación entre el valor económico de los recursos empleados y el valor económico de los resultados obtenidos, no entendida como al menor coste para el sistema, porque la factura la paga un solo grupo profesional.

Y para finalizar este segundo apartado dedicado a la prescripción enfermera, a las diferentes opciones que se nos presentan para su desarrollo, y a la postura que el COIB tiene respecto a la

\section{Cada vez se habla más de las atenciones para la salud en términos de eficiencia, y teniendo en cuenta que las demandas de salud son crecientes y los recursos son escasos, si la enfermera tuviera más instrumentos -como es el tema que abordamos hoy, es decir, la autonomía para prescribir determinados productos y/o medicamentos- podría resolver más situaciones de salud, y de esta manera estaríamos hablando de una enfermera más eficiente para el sistema de salud.}

prescriptor independiente" (el médico), ajustando dosis, modificándolas en función de la evolución del paciente o bien bajo protocolos consensuados o guías de práctica clínica, pero no puede considerarse prescripción independiente o autónoma de la enfermera pues sólo le permite prescribir dentro de los términos del protocolo, que ha consensuado o han sido supervisados por otro profesional.

En la actualidad estas 2 acciones se realizan en muchas ocasiones, sólo que no se reconoce formalmente. Su reconocimiento normalizaría una rutina que, efectivamente, hace posible agilizar o dinamizar la atención y, por lo tanto, el sistema de salud pero que a mi/nuestro juicio no está exenta de riesgos. Como reconocen los propios médicos cuando se refieren a su práctica profesional ${ }^{12}$, la aplicación de un protocolo-guía puede hacerla cualquier profesional-técnico, en ocasiones sin necesidad de ser ni médico ni enfermera, mientras que la aplicación adaptada a la situación del paciente concreto únicamente la puede hacer un profesional-experto, con un gran bagaje de conocimiento. dos o más partes"13, se convierte, en realidad, en "el supervisar", en el sentido de "ejercer la inspección superior en trabajos realizados por otros"13.

Cada vez se habla más de las atenciones para la salud en términos de eficiencia, y teniendo en cuenta que las demandas de salud son crecientes y los recursos son escasos, si la enfermera tuviera más instrumentos -como es el tema que abordamos hoy, es decir, la autonomía para prescribir determinados productos y/o medicamentos- podría resolver más situaciones de salud, y de esta manera estaríamos hablando de una enfermera más eficiente para el sistema de salud.

Siempre y cuando entendamos que eficiencia supone la correcta aplicación del cuerpo de conocimiento a la resolución de los problemas planteados por los clientes/usuarios/personas sujeto de nuestra atención, ya que la verdadera eficiencia, la que las enfermeras podemos conseguir, se producirá mayoritariamente en el ámbito de la prescripción autónoma, que es la que debemos reivindicar. En el ámbito de la denominada "prescripción colaborativa", la eficiencia que conseguiremos es la del sistema de salud. Como lo harán muchos prescripción, sólo me queda resaltar que yo soy de las que pienso que las enfermeras debemos afrontar el reto de la prescripción autónoma o independiente sin el menor asomo de miedo. Entre otras razones, porque estamos capacitadas para hacerlo y porque, si nos hace falta ayuda, nuestras corporaciones profesionales y científicas responderán. Y lo harán con todos los medios formativos e instrumentales necesarios para que esta habilidad -adquirida cuando estudiamos pero no desarrollada plenamente porque la ley nos lo ha impedido- la ejerzamos con toda seguridad, eficacia y responsabilidad.

Y también por 2 motivos más, que, a mi modo de ver, son definitivos: Porque ya lo estamos haciendo, y porque nadie está obligado a hacerlo. Es decir:

- Si lo estamos haciendo, o bien dejamos de hacerlo o asumimos del todo esta responsabilidad. No se puede admitir que a los responsables de las instituciones y/o empresas sanitarias ya les vaya bien que la enfermera prescriba en multitud de ocasiones pero "que no 
conste en ninguna parte", ni que las enfermeras asumamos parcialmente la responsabilidad. O peor aún, que queramos (o peor aún, celebremos) poder ejercerla sólo con el consentimiento de este otro profesional. - Si nadie está obligado a hacerlo, que se permita a cada enfermera que decida si asume esta responsabilidad. ¿O es que se puede obligar a un médico a recetar un medicamento? ¿O es que se puede obligar a un oftalmólogo a operar cataratas o a un cardiólogo a realizar un by-pass? Pues exactamente igual.

Pero si, por el contrario, no queremos este reconocimiento, estaremos impidiendo que las enfermeras, que sí necesitan de este instrumento y de esta oportunidad, por un lado resuelvan problemas a las personas y, por el otro, contribuyan al desarrollo profesional.

\section{LOS MOTIVOS PARA}

\section{EL "SÍ" Y LOS MOTIVOS PARA EL "NO" A LA PRESCRIPCIÓN ENFERMERA}

Finalizado el segundo apartado y para cumplir con el tercer objetivo

(profundizar algo más en esta capacidad prescriptora de la enfermera), que me servirá de conclusión, les propongo que me permitan "ponerme de moda", para resumirlo en términos de los motivos para el "sí" y los motivos para el "no" de esta prescripción.

\section{Por qué el "sí" a la prescripción enfermera}

Primera razón. Porque debemos dar la cara por aquello que es nuestro: cuestión de responsabilidad

Pero en aquel ámbito donde la enfermera es autónoma, en el desarrollo de la función de cuidar, en el basado en un modelo de cuidados donde las prescripciones que realiza la enfermera son de su exclusiva responsabilidad. Es aquí donde queremos el reconocimiento legal.

En el rol que ejerce basándose en su trabajo de colaboración con otros profesionales del equipo de salud y que incluye aquellas situaciones de salud de las personas que precisan diagnóstico y tratamiento médico, y donde la enfermera realiza actividades de vigilancia, control y prevención de complicaciones relativas a la situación de enfermedad y tratamiento médico que se ha establecido, es en el que hasta ahora nos "han dejado hacer". Medio a escondidas, actuando en los famosos "Nolotil, si precisa" o el "antitérmicos, si precisa" o "transcribiendo" la prescripción (por lo tanto la decisión de otro). Y que, en todo caso, se debería sólo reservar para cuando el problema que resolvemos es de la persona que atendemos o de quien actúa como cuidador/a principal. No el problema de otro profesional**

Pero, como ya he intentado argumentar suficientemente, esta prescripción no ha de ser la de interés principal de las enfermeras sino del sistema de salud. Y este ya ha dicho que no la quiere, y por esto es de muy difícil regulación. Por ello, también, considero que por lo que hemos de batallar es en el primer supuesto (en la autónoma). En la que no tan sólo si se indica un pañal determinado o la bolsa de ostomía más adecuada, o la pomada de "no sé qué", la enfermera no lo deba hacer "falsificando" la receta o entrando en cualquier sistema con el código de otro profesional. O pueda decidir si la pomada, o el antiséptico o el apósito de aquella herida es o el indicado o no.

Y yo, al menos, a esta prescripción (autónoma) no le tengo ningún miedo. A lo que sí le tengo miedo es a lo que yo no decido, o para lo que no tengo suficientes conocimientos o a lo que no forma parte de mi estricta responsabilidad profesional.

Y si un día se decide que es necesario discutir la denominada "prescripción colaborativa" (que a mí me gusta más denominarla "administración avanzada" o "prescripción en colaboración"), las enfermeras la asumiremos con los inconvenientes que siempre supone más carga de trabajo y más responsabilidad, siempre y cuando reviertan también en el profesional que la asuma (en este caso en la enfermera) los beneficios de la misma.

** Me refiero a que supongo que estaríamos de acuerdo -durante una visita domiciliaria- en evitar a cualquier cuidador/a principal acudir para cada receta al centro de salud, eliminando barreras de accesibilidad en poblaciones muy vulnerables y aportando más diligencia al conjunto de las intervenciones sanitarias. Como prescribir un analgésico, si se precisa, porque es la persona a quien prestamos nuestros cuidados quien lo necesita y no para evitar despertar a otro profesional.
Estos beneficios también son muchos: entre otros, el reconocimiento de la persona que atiende, el control del gasto, las ventajas de una determinada indicación o la productividad. Y no como sucede ahora, al menos en Cataluña, que, en este mar de confusión, las enfermeras cargamos con todas las desventajas (p. ej., la vacunación en la atención primaria, la protocolización y la estandarización en la hospitalaria y todas juntas -corregidas y aumentadas- en la sociosanitaria y social, que ya ha llegado a una situación de verdadera desesperación), mientras los beneficios de la misma los cobra otro profesional.

Segunda razón. Porque es necesario para nuestro ejercicio profesional Desde la incorporación de la diplomatura universitaria de enfermería, y, parece ser, la próxima titulación superior de grado, las enfermeras de nuestro país han visto que su papel va mucho más allá de unas funciones técnicas y delegadas y que abre las puertas a unas dimensiones muy amplias, que poco a poco han tomado forma y han supuesto para ellas un avance hacia niveles más óptimos de profesionalización, con un marco de competencias definido que ejerce de forma autónoma, tanto en el ámbito de actuación propio (a mí me gusta más denominarlo autónomo) como en el de colaboración.

Y lo que no se puede es aceptar que existan profesionales con estas competencias a la vez que se impide sistemáticamente su desarrollo, y tolerar que por otros motivos que nada tienen que ver con el servicio que prestan ni con el contenido técnico (competencias y capacidades) y el carácter ético (de compromiso y de respuesta a necesidades con la garantía de esta realización) del mismo que he nombrado al principio de mi intervención, sino con otras cuestiones como el poder de unos sobre los otros (clasismo) o la identificación de los cuidados con la función "natural" de la mujer (sexismo), por no citar algunas otras más.

No insistiré más en los beneficios de la prescripción enfermera (tanto la autónoma como la "en colaboración") más que para recordar que prescribir implica, frecuentemente, dar consejo sobre la medicación prescrita: uso, 
efectos deseados e indeseados, conservación y almacenamiento del producto. Y la educación sobre el uso del medicamento, que es una parte muy importante en el cuidado de las personas y que cuando la enfermera prescribe suele dar instrucciones muy precisas sobre el uso del producto, lo que disminuye el riesgo de incumplimiento terapéutico o de mala utilización.

\section{Tercera razón. Porque la enfermera} es una gran gestora de recursos

Esta característica, reconocida ya de manera general, puede ayudar, y mucho, a la eficiencia del sistema. ¿Porque ha sido formada para esto? Pues puede que sí, pero yo creo que principalmente porque forma parte de la naturaleza del cuidado y porque la enfermera no ha contado con estos recursos ni con el poder de decisión.

Y por otro motivo que está muy claro. Porque dentro del sistema de salud es quien da siempre la cara. Y cuando se sabe que tendrás que dar la cara, ya procuras evitar que "las cosas no funcionen bien". En parte porque así lo hemos querido las enfermeras pero, por otra, porque ha convenido que sea así. Por esto la enfermera es tan consciente de que los recursos son limitados y ha aprendido a gestionarlos bien. O cuando faltan sábanas, no hay toallas, no funciona la persiana, o la dieta no es adecuada, no hay el suficiente material, se retrasan las consultas, se suspende una intervención quirúrgica, etc., ¿cuál es la respuesta del médico o de la enfermera o de cualquier otro profesional?

No os hablaré de la experiencia de otros países, pese a que, como replica el dicho popular, "la experiencia es un punto" y siempre es bueno aprovecharla. Pero si una conclusión se puede sacar es que la experiencia de la prescripción enfermera en otros países ha sido un éxito.

Su puesta en marcha ha permitido mejorar la atención, demostrando que pueden prescribir de manera costeefectiva. El ahorro de tiempo, la continuidad de los cuidados, un mejor cumplimiento del tratamiento y las oportunidades de promoción de la salud aseguran un servicio más resolutivo y eficiente a los usuarios de los sistemas de salud y sociales y se ha mejorado en satisfacción y autonomía profesional.

\section{Por qué el "no" a la prescripción enfermera}

Primera razón. Porque esta prescripción no está estrictamente enmarcada en nuestro ámbito de competencia

El sistema sanitario no desea otra "normalización" de las habilidades y conocimientos de las enfermeras que la que asegura la realización de las técnicas derivadas del diagnóstico y tratamiento médico. Disponemos ya de muchos ejemplos y de otros tantos intentos de esta "normalización" (si entendemos por eso la confección de guías y de protocolos), que se hacen siempre basándose en a la disciplina médica. Por ejemplo, muchas técnicas complejas son definidas con criterios médicos. Y esto debe ser así. Pero entonces no hablemos de desarrollo profesional de la enfermera sino de contribución (sin ninguna compensación) a una parcial eficiencia de nuestro sistema de salud.

Me he referido extensamente a los motivos por los cuales debemos batallar en "nuestra porción". A mí me parece un error seguir batallando en otra porción. Lo he argumentado largamente. Pero en definitiva se resumen en 2: porque no es nuestro terreno, no es nuestra "porción", y batallar en porciones que pertenecen a otros es tener siempre las de perder, y porque privamos a los ciudadanos de otra de estas "porciones": la parte de atención que las enfermeras -y sólo nosotras- podemos proveer.

Aun reconociendo que quizás no tenemos más remedio que aceptar lo que de la nueva ley se derive (y parece que en este aspecto poco de nuevo derivará), mañana mismo debemos ponernos a trabajar para recuperar, simplemente, lo que es nuestro. Nadie, ni ninguna empresa sanitaria ni ninguna Administración, puede ni debe definir nuestras competencias. Esto sólo corresponde a la profesión.

\section{Segunda razón. Porque esta} prescripción autónoma no cuenta con un catálogo cerrado para su realización Sobre quien firma la receta recae la responsabilidad de la prescripción, y su emisión debe hacerse basándose en a una valoración y a un juicio clínico. Por eso es obvio que las prescripciones derivadas de la valoración y el juicio clínico de la enfermera deben ser firmadas por ésta, en el ejercicio de su competencia, y para ello es imprescindible disponer de un catálogo que limite -en este caso voluntariamente y en el mejor sentido de la palabralos productos y medicamentos de prescripción autónoma de la enfermera.

Y de sus ámbitos especialistas, es decir, los que corresponden a las matronas y a los especialistas en salud mental, que han de ver reconocidos las técnicas, los productos y los fármacos que constituyen instrumentos indispensables para su ejercicio profesional.

Tercera razón. Porque no se dispone del marco legal para desarrollarla

Las enfermeras pueden asumir responsabilidades en el ámbito de la prescripción farmacológica, pero el actual marco legal no lo permite. Aquello que no se puede hacer es llevar a término prácticas que resolverán y agilizarán la situación asistencial, pero que impliquen vulnerar la legislación vigente, con el consecuente riesgo para la responsabilidad personal y profesional de las enfermeras.

O se prescribe o no se prescribe, pero lo que de todos modos hay que evitar es que ahora las enfermeras "recetemos" lo que prescribe otro profesional

Y es más. Si hoy esta ley se aprueba sin la modificación, que parece que se ha abandonado, que consiste en la reformulación del antiguo artículo 76.1 (ahora 77.1) del proyecto de Ley de Garantías y Uso Racional de los Medicamentos y Productos Sanitarios donde dice: "La receta médica, pública o privada, y la prescripción hospitalaria son los documentos que aseguran la instauración de un tratamiento con medicamentos por instrucción de un médico o un odontólogo, únicos profesionales con la facultad para ordenar la prescripción de medicamentos y productos sanitarios", y donde pedíamos la sustitución de "únicos profesionales" por "profesionales principales", la situación de las enfermeras respecto a la prescripción 
(no enfermera, sino la actual)

empeorará***. Si esto ocurre, mi opinión

es que mañana mismo se vuelva a

plantear. Y en esta ocasión desde

la convicción irrevocable de que no hay

prescripción en colaboración (que

debería ser de interés del sistema

sanitario) si no hay prescripción

autónoma (que, seguro, es de interés

para la profesión enfermera).

\section{PARA FINALIZAR}

La prescripción enfermera es una competencia capital para contribuir en la eficacia de la gestión de los problemas de salud de la población, la gestión y optimización del uso de las prestaciones sanitarias, asistenciales y de cuidados aplicadas tal como demuestran múltiples estudios. También es determinante para el desarrollo del rol autónomo de nuestra profesión.

En la práctica cotidiana, la enfermera indica y utiliza medicamentos bajo criterio de buena práctica y juicio clínico, sin prescripción médica y sin disponer de reconocimiento de la competencia y de la autoridad legal, con el visto bueno, tácito o explícito, de las instituciones y la Administración sanitaria. Es preciso regular legalmente aquello que se está produciendo de facto. No se puede obligar a los profesionales a extralimitar el ámbito competencial legalmente establecido, sino que es necesario adaptar el marco legal a la realidad de la dinámica asistencial.

La enfermera tiene conocimientos suficientes, por su formación pregrado, para indicar y utilizar determinados medicamentos y productos sanitarios de

\footnotetext{
*** A la hora de la revisión de este artículo y, por lo tanto, con posterioridad a la conferencia pronunciada en el marco de la jornada "Prescripción Farmacológica Enfermera: Análisis de la situación y futuro", celebrada en San Sebastián, la modificación de este ariculo de la Ley de Garanis y Uso Racional de los de este ariculo de ha Ley de Garas y Uso Raci Medicamentos y Productos Sanitarios no se ha aprobado. Los servicios jurídicos del Col-legi Oficial d'Infermeria de Barcelona confirman que, a pesar de que desde un punto de vista de regulación jurídica no se puede considerar que la nueva ley signifique cambio alguno -por lo que a la prescripción enfermera se refiere- respecto a la vigente hasta este momento, el hecho de que se haya despreciado la oportunidad de el hecho de que se haya despreciado la oportunidad de
proporcionar acogida legal a determinadas prácticas vinculadas a la prescripción colaborativa de las enfermeras puede inducir a interpretar que la participación de las enfermeras en la prescripción de fármacos, que antes podía considerarse alegal o de dudoso amparo legal, ahora se considere claramente ilegalo contrana a la ley (en tanto que el legistad a cerrado la puerta a cualquier tipo de participación de los profesionales sanitarios que no sean médicos u odontólogos en el ámbito de la prescripción de fármacos).
}

forma autónoma. Por otra parte, entendemos que hace falta prever actividades de reciclaje y formación continua, o incluso la revisión del programa formativo de pregrado, especialidades o cursos de capacitación específica para el crecimiento de la mencionada competencia, para ajustarse día a día a nuestra realidad. Yo estoy segura de que nuestras organizaciones colegiales así lo harán. El compromiso de Barcelona aquí está.

La posibilidad de prescribir determinados fármacos y productos significa una gran mejora en la atención que reciben los ciudadanos y las ciudadanas y para el desarrollo de la práctica enfermera. Los medicamentos y productos que puede prescribir la enfermera deben ser los derivados de su práctica y ámbito competencial. No se trata de invadir competencias de otros profesionales que tienen su propia actividad y responsabilidad de prescripción y recetación. Hace falta aprobar una lista de medicamentos y productos sanitarios -y con esto estamos trabajando- sobre los que la enfermera tenga potestad de prescripción, modificando las leyes correspondientes.

Querría citar de nuevo a esta enfermera a la que he hecho referencia, que en uno de sus muchos artículos recuerda que "en los últimos 20 años, las enfermeras españolas hemos conseguido un mundo. Ahora lo que toca es habitarlo; o sea, poseerlo y utilizarlo con orgullo, confort y alegría"3.

Qué cierto es. Pero permítanme de todos modos que yo añada que conozco y puedo dar fe de que existe un gran número de enfermeras y enfermeros dispuestos a ello. Que tienen los conocimientos, la fuerza y la voluntad para ello. Ahora sólo les hace falta la confianza para que puedan hacerlo.

Y las corporaciones que tenemos el honor de representarlas y representarlos, la obligación de luchar por ello.

\section{BIBLIOGRAFÍA}

1. Cuxart Ainaud N. Instrumentos para cuidar Estrategias de implantación. Nursing (Barcelona). 2003;21(7):55-61.

2. Alberdi RM. La identidad profesional de la enfermera. Revista ROL de Enfermería. 1992;170:39-44.

3. Alberdi Castell RM. La formación superior como instrumento para el desarrollo de la Enfermería. Revista ROL de Enfermería. 2000;23:99-103.

4. Alberdi Castell RM. Enfermeros, buscando respuestas. Conferencia de las jornadas organizadas por la Asociación Española de Enfermería Docente (AEED) y los Hospitales del SAS. Jerez, 9 y 10 de noviembre de 1995. No publicada.

5. Wolinsky FD. The professional dominance perspective revisited. The Milbank Quarterly. 1988;66 Suppl 2:33-47. Citado en Pardell H. ¿Tiene sentido hablar de profesionalismo hoy? Disponible en: http://www.smu.org.uy. Consultado el 22 de junio de 2006

6. Alberdi Castell RM. Las enfermeras del futuro: propuesta para la excelencia profesional. Revista de la Asociación Española de Enfermería en Urología. 1999:73:17-23.

7. Alberdi Castell RM. Estrategias de poder y liderazgo para desarrollar el poder de las enfermeras. Revista ROL de Enfermería. 1998;239-240:27-31.

8. Fernández Ferrín C. La investigación en cuidados y el desarrollo disciplinar. En: Libro de las XVIII Sesiones de Trabajo de la Asociación Española de Enfermería Docente. Cádiz: Asociación Española de Enfermería Docente; 1997; p. 441-57.

9. Guy ME. Professionals in organizations. Citado en Pardell H. ¿Tiene sentido hablar de profesionalismo hoy? Disponible en: http://www.smu.org.uy. Consultado el 22 de junio de 2006

10. Organización Colegial de Enfermería: Consejo General. Prescripción enfermera. Análisis de situación y acciones a emprender. Septiembre de 2005. Disponible en: http://www.actualidad.enfermundi.com. Consultado el 16 de enero de 2006.

11. Organización Colegial de Enfermería: Consejo General. Marco referencial para la prescripción enfermera. Documento base elaborado por el Consejo General de Enfermería y presentado a los medios de comunicación el 23 de marzo de 2006. 12. Pardell H. ¿Tiene sentido hablar de profesionalismo hoy? Disponible en: http://www.smu.org.uy. Consultado el 22 de junio de 2006.

13. Real Academia Española. Disponible en: http://buscon.rae.es/draeI/. Consultado el 20 de junio de 2006.

\section{BIBLIOGRAFÍA COMPLEMENTARIA}

Alberdi Castell RM. Los contextos conceptuales históricos: una propuesta de análisis de la evolución de la profesión enfermera desde los orígenes hasta el siglo XIX. Revista Híades. 1998;5-6:63-73.

Alberdi Castell RM. Las enfermeras para el tercer milenio. Revista ROL de Enfermería. 1993;178:43-50. Alberdi RM. La ética del cuidado. En: Bosch E, Ferrer VA, Riera Y y Alberdi RM, editores. Feminismo en las aulas. Palma de Mallorca: Universitat de les Illes Balears; 2003. p. 160-88. Cuxart Ainaud, N. La presencia enfermera en el siglo XXI. Publicado en formato digital. Disponible en: http://www.coib.org. Consultado el 20 de junio de 2006.

Cuxart N, Alberdi Castell RM, Cuxart Ainaud N. Cuidados, enfermeras y desarrollo profesional: Una reflexión sobre las bases del ejercicio profesional. Presencia. 2005;1(2). Disponible en:

http://www.index-f.com/presencia/n2/23articulo.php Consultado el 20 de junio de 2006.

Ley 44/2003, de 21 de noviembre, de ordenación de las profesiones sanitarias (artículo 9).

Correspondencia: N. Cuxart Ainaud.

Col-legi Oficial d'Infermeria de Barcelona.

Alcoi, 21. 08022 Barcelona.

Correo electrónico: ncuxart@coib.org 